\title{
INVESTIGACIONES
}

\section{Motricidad escolar: Reflexiones y acciones en una experiencia situada en la educación básica}

\author{
Motricity at school: Reflections and actions in an experience situated \\ in elementary school
}

\author{
Motricidade escolar: Reflexões e ações em uma experiência \\ situada no ensino fundamental
}

\begin{abstract}
Clayton da Silva Carmo, ${ }^{a}$ Luiz Gonçalves Junior, ${ }^{b}$ José María Pazos $^{c}$

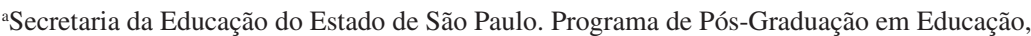
Universidade Federal de São Carlos, Brasil. Sociedade de Pesquisa Qualitativa em Motricidade Humana. Correo electrónico: spina002@gmail.com

bepartamento de Educação Física e Motricidade Humana, Programa de Pós-Graduação em Educação, Universidade Federal de São Carlos, Brasil. Sociedade de Pesquisa Qualitativa em Motricidade Humana. Correo electrónico: luiz@ufscar.br

'Universidad de Vigo, España. Correo electrónico: chema3@gmail.com
\end{abstract}

\begin{abstract}
RESUMEN
Este trabajo es producto de una actuación en el campo de la Educación Física Escolar, orientada por referentes teóricos de la Motricidad Humana y de la Pedagogía Dialógica. Así comprendiendo la experiencia educativa como fenómeno complejo y situado, desarrollamos un proyecto de intervención en sesiones de Educación Física en una escuela de enseñanza básica, lo cual consistió en un conjunto situaciones de aprendizaje desarrolladas junto a un profesor de Educación Física. Consideramos que la experiencia desafió tanto a los niños como a nosotros educadores a presentar respuestas a las cuestiones que emergían de esas situaciones, proporcionando ejercicio de autonomía, de convivencia y de construcción de conocimiento, siendo algo fundamental para comprender y buscar la transformación de las prácticas que, habitualmente reproducidas, se hacen alienadas, vacías de sentido.

Palabras clave: educación física, motricidad humana, pedagogía dialógica.
\end{abstract}

\section{ABSTRACT}

This study is the result of an intervention in the field of Physical Education and is guided by some re-known theorists of the Human Motricity and Dialogic Education fields. Thus, understanding the educational experience as a complex and situated phenomenon, we developed an intervention project in Physical Education classes in an elementary school which consisted of a set of learning situations accompanied by a Physical Education teacher. We believe that the experience challenged both students and educators to provide answers to the questions that emerged from the situations, allowing an exercise of autonomy, coexistence and construction of knowledge as fundamental to understand and seek the transformation of the practices that reproduced as usual become alienated and empty of meaning.

Key words: physical education, human motricity, dialogic pedagogy.

\section{RESUMO}

Este trabalho é produto de uma ação no campo da Educação Física Escolar, sendo orientada por referenciais teóricos da Motricidade Humana e da Pedagogia Dialógica. Assim, compreendendo a experiência educativa como 
fenômeno complexo e situado, desenvolvemos um projeto de intervenção em aulas de Educação Física em uma escola de ensino fundamental, a qual consistiu em um conjunto de situações de aprendizagem desenvolvidas junto a um professor de Educação Física. Consideramos que a experiência desafiou tanto aos estudantes como aos educadores a apresentar respostas para as questões que emergiram dessas situações, proporcionando exercício de autonomia, de convivência e de construção de conhecimento, sendo algo fundamental para compreender e buscar a transformação das práticas que, habitualmente reproduzidas, se fazem alienadas, esvaziadas de sentido.

Palavras chave: educação física, motricidade humana, pedagogia dialógica.

\section{INTRODUCCIÓN}

El presente trabajo ${ }^{1}$ es producto de una actuación, llevada a cabo principalmente en el campo de la Educación Física Escolar, orientada por referentes teóricos de la Motricidad Humana y de la Pedagogía Dialógica, por eso optamos por iniciar con una breve contextualización sobre los mismos.

La Motricidad Humana puede ser comprendida erróneamente como sinónimo de movimiento. Decimos esto porque el movimiento puede estar presente en diversas situaciones, por ejemplo una hoja de papel al viento se mueve, y este es muy diferente de los movimientos realizados por una persona; por eso, cuando se trata de motricidad humana lo que tenemos es acción, no movimiento, y la hoja de papel no posee ninguna acción en el movimiento que podemos percibir.

La Motricidad Humana comprende, segundo Sérgio y Toro (2005), no sólo una definición pertinente de un objeto de estudio que le permita desarrollarse desde el punto de vista científico, sino también define al observador que se acerca y analiza y vive con el objeto, vale decir nos encontramos ante una acción humana que no se separa de su actor, sino que constituye una extensión del mismo y, por lo tanto, también actúa sobre él mismo.

El término Motricidad Humana es propuesto por Manuel Sérgio (1991) y es comprendido como la energía (intencionalidad) para la acción humana en el mundo, o sea, es la intencionalidad que en la acción se corporeiza, se expresa. Cuando adoptamos esta perspectiva epistemológica en nuestra intervención, buscamos, en acuerdo con Sérgio (2003), la superación de la Educación Física que, llevada a cabo bajo la visión fragmentada del ser humano, destina al cuerpo únicamente la valoración del desarrollo físico y de los ejercicios orientados a la salud en su sentido estrictamente bio-fisiológico, y planteamos el salto del paradigma cartesiano al paradigma de la complejidad: el paso de la Educación Física a la Ciencia de la Motricidad Humana (CMH). Por ese motivo nosotros, miembros de la Sociedad de Investigación Cualitativa en Motricidad Humana, optamos por utilizar la expresión "Motricidad Escolar" en lugar de Educación Física, aunque aún mantengamos la denominación debido a las implicaciones legales que denominan el área y el componente curricular dentro de las escuelas.

La Pedagogía Dialógica, también conocida como pedagogía libertadora, es la propuesta pedagógica asumida por Paulo Freire (2005) para contraponerse a los modelos educativos pautados en la práctica bancaria, o sea, aquella en la que los estudiantes parecen cuentas en las que los profesores realizan ingresos constantemente y, al final de determinados periodos, sacan extractos (exámenes) para averiguar los rendimientos. Optamos por la nomenclatura

Texto adaptado de la tesis de magister de Carmo (2013), la cual contó con apoyo de la "Coordenação de Aperfeiçoamento de Pessoal de Nível Superior" (CAPES-Brasil). 
dialógica, pues en esa propuesta el diálogo tiene un papel central en el desarrollo del proceso educativo, y en la perspectiva presentada, la postura dialógica es fundamental en cualquier acción que se pretenda verdaderamente educativa. En ese sentido, la comprensión que hemos adoptado de educación y que orientó nuestras acciones durante el proceso de intervención, propone que los educadores y educadoras cambien el "hablar para” por "hablar con" (Freire, 2005).

Merleau-Ponty (2006) cuando trata el papel del cuerpo humano en los estudios de la naturaleza, dice que no es una simple cosa, y que es en sí una relación con el mundo, es un cuerpo que se mueve, que percibe y que se percibe. Según el autor, es necesario tener el cuerpo como el sujeto del movimiento y de la percepción, y no como un cuerpo-objeto en el cual la conciencia actúa y habita. El cuerpo es empatía con el mundo, con las cosas y con otros cuerpos (intercorporeidad).

En este sentido, y comprendiendo la experiencia educativa como un fenómeno necesariamente complejo y situado, en el año 2011, desarrollamos un proyecto de intervención en sesiones de Educación Física en una escuela de enseñanza básica en la red estatal pautados en los citados referenciales. La escuela se localiza en la región central del municipio de São Carlos, São Paulo, Brasil. La intervención consistió en un conjunto de seis situaciones de aprendizaje desarrolladas en trece sesiones impartidas, junto a un profesor de Educación Física de la escuela, para una clase de $4^{\circ}$ curso de enseñanza básica.

Antes de iniciar las sesiones realizamos una investigación temática. Esa investigación, como propone Freire (2005), debe ser el punto de partida para cualquier acción educativa, pues es el momento en que, junto a las demás personas envueltas en el acto educativo, el/la educador/a identifica los temas generadores, o sea, las temáticas significativas originarias de las experiencias cotidianas del grupo que se presentan como imperativas al desarrollo de la acción pedagógica. Así, los contenidos programáticos emergen para el/la educador/a de un contexto situado y por eso reflejan los anhelos y esperanzas de las personas envueltas.

En ese proceso de investigación buscamos dialogar con los niños de la clase, sus padres, madres o responsables, con el Profesor de Educación Física, la Profesora de la clase y también con el restante personal de la escuela (Dirección, Coordinación, Secretaría, Limpieza, Cocina). Con estos últimos tuvimos conversaciones individuales en las que les preguntamos sobre temas que consideraran importantes para tratar con los niños de la clase en la que estábamos interviniendo. Ya con los padres, madres y responsables el cuestionamiento fue realizado en la reunión de padres y maestros de la clase, en el que hicimos una sensibilización de los presentes para exponer el proyecto de actuación en base a la motricidad humana y a la pedagogía dialógica. En esa reunión presentamos fotos y videos de actividades realizadas por los niños en el semestre anterior, aproximando a los familiares a las actividades desarrolladas en Educación Física y discutiendo con ellos los próximos pasos, cuestionando qué temáticas deberían ser desarrolladas en las próximas sesiones de aquel grupo. En esa ocasión no estaban presentes todos los responsables, por eso elaboramos una carta de presentación del proyecto y la enviamos juntamente con las cuestiones que orientaban la investigación temática a fin de permitir, de alguna manera, que las personas que no pudieron estar presentes en la reunión participaran y contribuyeran con la temática de la investigación. Las cuestiones enviadas por carta fueron las siguientes: 1) En su opinión de padre, madre o responsable ¿que tema/asunto es importante que los niños aprendan en la escuela?, 2) ¿Cómo pueden contribuir a ello las sesiones de Educación Física? 
Al final de la investigación temática, después de lecturas y relecturas de los registros, surgieron dos temas principales que fueron abordados en la intervención, a saber: "Respeto" e "Historia de los juegos y deportes". El primer tema tuvo gran relevancia, principalmente en las sugerencias de familiares y funcionarios, y el segundo surgió principalmente del diálogo con niños, en el cual la práctica de diversos juegos y deportes predominó, así como el interés por el origen histórico de esas prácticas.

Ante eso, iniciamos el proceso de problematización de los temas con los niños durante las clases de Educación Física. La problematización es el momento en que las personas envueltas en el acto educativo direccionan sus reflexiones sobre una situación concreta, promoviendo una percepción crítica en pro de la realidad objetiva con el fin de avanzar para transformarla, pues las personas “[...] son porque están en situación. Y serán tanto más en cuanto no solo piensen críticamente sobre su forma de estar, y actúen críticamente sobre la situación en que están" (Freire, 2005, p. 116).

En el caso de la acción educativa aquí presentada, buscamos realizar la problematización a través de la utilización de lo que llamamos actividades generadoras. Estas son las actividades que organizamos con intención de problematizar los temas generadores, pudiendo estas desencadenar la necesidad de investigar otros temas, y la realización de otras actividades tanto para el estudio de otros temas, como para profundizar en un tema ya en estudio.

En clase, los dos temas elegidos se fueron desarrollando en cierta medida, de modo simultáneo, buscando el establecimiento de respeto mutuo durante el desarrollo de las actividades citadas, pues es necesario para el aprendizaje del grupo. Así, cuando estábamos en clase estudiando la Historia de los juegos y deportes, también estudiábamos el Respeto debatiendo, principalmente, las situaciones de falta de respeto que sucedían en las actividades, relacionándolas con las situaciones similares ocurridas en los deportes y problematizando colectivamente los motivos y buscando una posible solución.

Todas las actividades realizadas fueron elaboradas considerando las voces de los niños y familiares durante a investigación temática y también surgieron del contexto de desarrollo de las clases.

Presentamos a continuación las situaciones de aprendizaje utilizadas en las clases, algunas de ellas compuestas por más de una sesión, por eso tienen una descripción general de la situación de aprendizaje y, al final de cada descripción, un breve relato que contiene nuestras principales reflexiones sobre cada una de ellas, y también de las acciones desencadenadas a partir de estas, para que el/la lector/a pueda, mínimamente, acompañar los procesos educativos desencadenados a lo largo de cada una de las sesiones.

\section{SITUACIÓN DE APRENDIZAJE 01: LA CREACIÓN DE UN JUEGO (SESIONES DE 1 A 3)}

Para la realización de esta actividad se dedicaron tres sesiones. Consistió en proponer la creación de un juego a partir de materiales predeterminados puestos a disposición de los niños, a saber: tapas de botella PET y bastones de cubo de basura de aproximadamente 40 $\mathrm{cm}$. Con los niños divididos en grupos de cinco personas, les pedimos que crearan un juego nuevo utilizando esos materiales; podían utilizar solo las tapas, solo los bastones o ambos, la decisión quedó a criterio de cada grupo. La opción de materiales alternativos mostró un 
distanciamiento de materiales deportivos que fácilmente remitirían a deportes mediáticos y posiblemente limitaría el proceso de creación, recayendo en la reproducción simple. Con los grupos finalizando el proceso de creación pasamos a la fase de presentación y vivencia, en el que cada grupo presentaba su juego y todos lo vivenciaban. Después de cada vivencia dialogábamos sobre las similitudes y diferencias entre los juegos creados por nosotros y los deportes y juegos ya existentes y también entre los propios juegos que conocemos. Se encontraron similitudes en los movimientos de acercamiento, de separación y diferencias en relación al implemento utilizado por nosotros y los utilizados en otras prácticas.

El Respeto fue el tema base de la actividad en la organización colectiva en la que buscamos pasar frecuentemente por los grupos auxiliando y orientando, cuando es necesario en la resolución de los conflictos ${ }^{2}$, en la coordinación y también en la construcción de los juegos. Surgieron diversos conflictos de esa construcción colectiva, entre ellos podemos citar la relación entre los niños en los grupos y también la dificultad de estos a desvincularse de los deportes hegemónicos.

\section{REFLEXIONES SOBRE LA PRÁCTICA Y ACCIONES DESENCADENADAS}

\section{Sesión 01}

Reflexiones: El trabajo en grupo, en parte, fue perjudicado por el descontento de los niños con relación a la división aleatoria que hicimos. Eso quedó bastante evidente en uno de los grupos en que solo había quedado una niña, la cual quiso cambiar e insistimos para que ella se quedara, pues estábamos preocupados en mantener niños y niñas en todos los grupos. Llegamos a la conclusión de que crear nosotros los grupos no promueve la interacción entre géneros y, además de eso, asumimos la responsabilidad de una tarea que ciertamente puede ser de los niños. Tuvimos también mucha dificultad en la realización del trabajo en grupo, pues los niños, aún estando en los grupos trabajaban individualmente, o sea, no dialogaban entre ellos en la tentativa de crear un juego. Cada estudiante buscaba crear algo solo o con un amigo, cuando por coincidencia pertenecían al mismo grupo, en pocos momentos sentimos que los grupos se reunían para pensar colectivamente sobre la actividad.

Acciones: Para las sesiones siguientes decidimos dejar la división de los grupos a cargo de los niños, solamente estableciendo parámetros del número de personas y distribución equitativa de género entre los grupos y acompañar el máximo posible a las actividades en grupo, para orientar a los niños en sus elecciones e intentar posibilitar la participación de todos.

\section{Sesión 02}

Reflexiones: La dificultad del trabajo en grupo también emergió en la segunda sesión en uno de los grupos, pues dos niñas que habían faltado a la clase en la que fueron creados los juegos, entraron en uno de los grupos y, sin que nos diéramos cuenta, cambiaron el juego de acuerdo con sus intereses sin consultar al resto del grupo que ya había inventado otro juego en la sesión anterior; sin embargo sólo percibimos eso al final con el desencadenamiento del conflicto entre los integrantes durante la vivencia del juego propuesto por las citadas niñas del grupo.

De acuerdo con Jares (2002), el conflicto se ha asociado normalmente a algo negativo, sinónimo de violencia, el carácter de algo no deseable, sin embargo ello posibilita las transformaciones. Convivir requiere aprender a conducir los conflictos y tensiones emergentes de ellos a un resultado positivo, generador de conocimiento y no de violencia, que aparece cuando situaciones de conflicto no son efectivamente problematizadas. 
Acciones: Sobre el conflicto con el cambio del juego realizado por las niñas, decidimos dialogar al final de la sesión sobre el respeto necesario para el trabajo en grupo y para valorar y demostrar respeto al trabajo realizado anteriormente por el grupo, nosotros combinamos colectivamente que vivenciaríamos en la clase siguiente el juego originalmente construido por el grupo, a saber: a Queimada Bastampa ${ }^{3}$.

\section{Sesión 03}

Reflexiones: La auto-organización de los niños en la división de los equipos reveló conflictos, lo que nos permitió una mejor comprensión de las relaciones de poder que estaban establecidas entre ellos y que indicaban la necesidad de mucha orientación y mediación en los conflictos para superarlos. Percibimos claramente que, dividir los equipos aleatoriamente intentando evitar los conflictos comunes al proceso, niega a los niños la responsabilidad y el cuidado que deben tener unos con los otros en ese contexto.

A los niños les gustó bastante el juego Queimada Bastampa presentado por el grupo en esa sesión, sin embargo el tiempo de clase fue poco por la necesidad de resolver los conflictos originados con la creación de los grupos. Ello originó una discusión, realizada al final de la clase, que ocupó mucho tiempo y lo restó al trabajo colectivo realizado por los compañeros de ese grupo que habían sido dejados de lado, que acabó siendo bastante breve para desarrollar las actividades.

Acciones: Estar más atento a la relaciones establecidas en los grupos y entre ellos en las próximas sesiones.

\section{SITUACIÓN DE APRENDIZAJE 02: LA TRANSFORMACIÓN DE UN JUEGO (SESIONES DE 4 A 6)}

Para esa situación fueron dedicadas tres sesiones. Consistió en la modificación de uno de los juegos creados por la clase con la intuición de vivenciar el proceso de transformación que las prácticas deportivas y los juegos sufren históricamente al difundirse en diferentes contextos y que, a veces, generan prácticas bastante diferentes. Así, después de la selección conjunta de uno de los juegos anteriormente elaborados, iniciamos el proceso de transformación. El juego escogido consistía en tres equipos: dos quedarían en los extremos de la pista, uno de cada lado, atrás de la línea de fondo de la pista de vóley, y la tercera quedaría dentro de la pista como lanzadora. Anotaba punto quien consiguiera atravesar la pista y tocar la tapa del otro lado. El juego terminaba cuando los equipos no tuvieran más personas para ser elegidas, pues estas deberían salir, o cuando uno de los equipos de los extremos consiguiera anotar cinco puntos.

Con los niños, consensuadamente divididos en dos grupos: Reino de Mulamba y Reino de Ubuntu ${ }^{4}$, iniciamos el proceso de modificación colectiva del juego, así cada grupo representaba un reino, un país, en que aquel juego había llegado y después, con el pasar de

\footnotetext{
El juego se asemejaba a un juego de quemada tradicional, sin embargo, en el lugar del balón se utilizaba una tapa de botella PET y, para lanzarla, se utilizaba un bastón de madera. Quién cogiera la tapa tendría el derecho de relanzarla y para eso iría hasta a lateral de la manzana a coger el bastón. Tras relanzar, el jugador debería devolver el bastón. Lo mismo sucedería con los jugadores del muerto. Quién fuera quemado debería ir para el muerto. La tapa debería ser relanzada en el suelo y cada persona tendría tantas oportunidades como fueran necesarias para conseguir relanzar.

4 Nombres ficticios sugeridos por el profesor de Educación Física del grupo.
} 
los años y las modificaciones que las personas hicieron, cada reino acabó teniendo un juego distinguido, aun teniendo originalmente el mismo juego como base. Conversamos sobre los motivos que tienen los personajes y debatimos también la relación de algunos juegos con el periodo de cosecha o, aun, con festividades religiosas.

Con los grupos trabajando en la transformación, fuimos pasando y ayudando en la organización y elaboración del juego cuestionando el tiempo de espera para las personas que quedaban fuera y también sobre la exclusión de las personas durante la actividad, cuando eventualmente alguien sugería cambios que caminaran en ese sentido.

El Respeto compuso la actividad principalmente por medio de la orientación y mediación del proceso de construcción colectiva, con intervenciones en el sentido de promover la participación de todos los niños presentando sus sugerencias, dudas y discordancias y la búsqueda de consenso y de enfoques del juego. También fueron importantes las demandas realizadas por los grupos exigiendo que algunos de los niños, que no colaboraban con el trabajo que estaba siendo realizado, asumieran parte de la responsabilidad del proceso de construcción colectiva del grupo. Esos momentos exigieron nuestra mediación para evitar que tales conflictos se desencadenaran en actitudes de exclusión o aislamiento.

En las dos sesiones siguientes, después de retomar la conversación, el juego original y el proceso de modificación colectiva realizado por los grupos, vivenciamos respectivamente los juegos modificados por los reinos de Mulamba y de Ubuntu, estos tuvieron las debidas explicaciones y presentaciones de las reglas realizadas por integrantes de cada grupo responsable. Después de la vivencia de los juegos dialogamos con los niños sobre el proceso de modificación de las reglas con la intención de problematizar y comprender mínimamente el proceso de transformación de juegos y deportes conocidos actualmente, así también observamos las semejanzas entre juegos de orígenes distinguidos y diferencias en juegos de mismo origen practicados en diversas regiones.

\section{REFLEXIONES SOBRE LA PRÁCTICA Y ACCIONES DESENCADENADAS}

\section{Sesión 04}

Reflexiones: Una fuerte lluvia redujo la presencia de los niños y no hizo viable el uso del área externa. El trabajo en grupo con un grupo más pequeño facilitó el acompañamiento y nuestras orientaciones frecuentes a los grupos, lo que fue importante para estimular la participación de todos los niños en el ejercicio de construcción colectiva. Creemos que la división de grupos también fue favorecida por el bajo número de alumnos/las, que esta vez no presentó ningún conflicto. Sin embargo quedamos preocupados, pues la falta de gran parte de los niños podría comprometer la marcha de la actividad.

Acciones: Retomar todo el proceso en el inicio de la próxima sesión, describiéndolo, para que los niños que faltaron entiendan el qué y por qué fueron hechos tales cambios, antes de vivenciar los juegos.

\section{Sesión 05}

Reflexiones: Un niño, que participó de la elaboración de los juegos en la sesión anterior, nos preguntó si íbamos a jugarse fútbol. Los niños, inclusive este niño, hicieron diversas sugerencias distintas del fútbol y asumieron que sería interesante conocer la historia de los juegos y deportes, sin embargo, comenzaron a surgir sugerencias a favor de la práctica del fútbol. La organización de los equipos bajo la responsabilidad del alumnado generó 
conflictos, lo que limitó el tiempo destinado al juego, pues algunos niños más influyentes estaban escogiendo sus propios equipos, lo que excluía a otros.

Acciones: En el caso del fútbol, preguntamos al niño qué actividad habíamos acordado en grupo para realizar en esa clase y, con la respuesta, el mismo comprendió, aunque sin quedar plenamente satisfecho, que realizaríamos la propuesta acordada y participó de la actividad normalmente. Sobre la división de los equipos tratamos durante la sesión el proceso para garantizar un reparto consensuado. Para la próxima sesión debemos explicar mejor, desde el principio, lo que significa división colectiva de los equipos.

\section{Sesión 06}

Reflexiones: La división de los equipos fue realizada por los propios niños, esta vez sin indicaciones respecto a que tuvieran integrantes de los dos géneros, y tal vez hasta a causa de ello los conflictos fueron menores que en la sesión anterior. Fue interesante notar que, después de todas las negociaciones entre los niños, todos los grupos formados eran mixtos. Uno de los alumnos insistió en la solicitud de jugar al fútbol, que ya había sido hablada en sesiones anteriores, estábamos preocupados y ante la duda comenzamos a tener la impresión de que los niños no sabían bien lo que estaban haciendo aún con ellas participando de las discusiones y problematizaciones sobre los juegos. Esta duda se confirmó al final de la sesión, cuando preguntamos a ellas porque estaban haciendo aquellas actividades y ellas no supieron responder.

Acciones: Recordamos al final de la sesión que ellos habían demostrado el interés en el origen de los juegos y deportes y que estábamos haciendo esas actividades para entender cómo surgen los mismos, se transforman, se difunden por el mundo. Solicitamos que dijeran la historia de los deportes y juegos que les gustaría conocer y establecimos colectivamente las siguientes modalidades: Baloncesto, Voleibol, Fútbol y Bocha, esta última fue una sugerencia de uno de los padres y que presentamos a fin de posibilitar el conocimiento de la nueva modalidad. Mantuvimos la auto-organización sin restricción de género en las siguientes sesiones.

\section{SITUACIÓN DE APRENDIZAJE 03: RECONSTRUYENDO EL BASQUET (SESIONES DE 7 A 9)}

Realizadas también en tres sesiones, esta situación de aprendizaje tuvo como objetivo una reconstrucción de la modalidad deportiva baloncesto junto a los niños. Partiendo de una actividad conocida como Balón a la torre, ${ }^{5}$ problematizamos el origen del juego y contextualizamos la situación de creación de este en la Asociación Cristiana de Jóvenes con la necesidad de creación de un juego que pudiera ser realizado en un patio cubierto, pues en Estados Unidos, en el invierno, la práctica del fútbol era inviable. Dialogamos recordando las sesiones en que habíamos creado algunos juegos y comentando que así como nosotros, ellos elaboraron el juego a partir de materiales disponibles como balón de fútbol, cesto de madera usados en la cosecha de durazno y el espacio cubierto que poseía, inclusive, columnas en medio del espacio de juego. Con esa actividad buscamos facilitar la

Juego similar al baloncesto que tiene como cesta una persona de pie sobre una silla y permite incluir más participantes. El juego no permite caminar botando el balón, este se desplaza por el campo solo por los pases, es comúnmente utilizado como juego pre-deportivo de la citada modalidad, sin embargo en el caso no fue nuestra intención. 
comprensión del proceso de creación de modalidades ya instituidas y también enfatizar la posibilidad y, eventualmente, necesidad de reinventarlas.

El juego empezó con el grupo dividido en dos grandes equipos (16 personas) para practicar el juego balón torre, utilizando como balón un saco con tapas en el patio cubierto. A partir de esa situación inicial que poseía gran número de participantes, en un espacio no delimitado y un balón inadecuado, fuimos cuestionando algunos cambios que ocurrieron en la modalidad deportiva en cuestión, tales como la confección de un balón específico para la modalidad, la delimitación del espacio, la restricción del número de participantes y la confección de un cesto sin fondo para facilitar el juego. Debatíamos cada sugerencia hecha por los niños y después de acordar los cambios necesarios vivenciábamos el juego conforme a lo indicado, haciendo nuevas alteraciones para mejorar la dinámica del mismo.

El Respeto fue trabajado en la medida en que en la organización y mediación de las actividades buscábamos garantizar un espacio favorable al diálogo, eliminando las descalificaciones destinadas a algún niño, favoreciendo una participación activa de todos. La cuestión del Respeto también fue bastante abordada en los momentos de división de los equipos, y algunos conflictos tuvieron que ser mediados para que consiguiéramos despertar una solución pacífica. Además de eso, también ocurrieron muchas intervenciones y conversaciones sobre el equilibrio en la elección de las personas para que los equipos quedaran equivalentes evitando desestimular aquellos que estaban aprendiendo. Así, en momentos en que ocurrían mini juegos simultáneos, frecuentemente mediábamos el cambio de jugadores de equipo para promover la equidad entre los equipos y favorecer participación de todos/as.

Durante los juegos orientamos a los niños con más dificultad y por algunas veces paramos la actividad para acordar la necesidad de respeto a los compañeros, evitando actitudes violentas al coger el balón y también combatiendo la exclusión de los menos habilidosos, esas cuestiones también fueron problematizadas en la conversación final que fue realizada en cada una de las aulas. Fue necesario también enfatizar al grupo la responsabilidad de cada uno en el proceso de organización del aula, en el establecimiento del respeto para con las otras personas, sean ellas profesores/las o compañeros, y también la disciplina necesaria a la actividades.

\section{REFLEXIONES SOBRE LA PRÁCTICA Y ACCIONES DESENCADENADAS}

\section{Sesión 07}

Reflexiones: En esa sesión no realizamos la división auto-organizada por los niños, pues nuestro foco principal era la problematización del juego de baloncesto y el tiempo iba a ser insuficiente para la realización de las dos actividades. Durante la vivencia del juego, la marginación de las niñas y de los menos habilidosos quedó evidente, así como el aumento de la violencia en las actitudes de los niños.

Acciones: Debatimos un poco la cuestión de la violencia al final de la primera sesión y pedimos algunas sugerencias para los niños, de entre las cuales quedó acordado para la siguiente clase la disminución del número de personas en los equipos. Debatimos también brevemente sobre los cambios ocurridos en las modalidades deportivas y sobre la violencia observada en ellas en las transmisiones por la televisión. Para la sesión siguiente decidimos debatir la cuestión del Respeto más enfáticamente durante la realización de los mini juegos de baloncesto debido a las últimas observaciones realizadas. 


\section{Sesión 08}

Reflexiones: El grupo presentó un comportamiento que hacía difícil la organización de la actividad prevista. Muchos niños pedían que gritáramos pidiendo que los otros pararan de hacer barullo. Insistimos diciendo que era necesario un mínimo de organización para hacer las actividades y la conversación con los niños se demoró bastante, pero consiguió algún efecto. Algunos niños vinieron a negociar con nosotros la posibilidad de jugar al fútbol libremente en mitad de la sesión, sin embargo insistimos recordando lo que había sido acordado en la sesión anterior. Al intentar iniciar la división de los equipos con los niños fue extremadamente evidente que la habilidad, género y hasta la apariencia de las personas condicionan las elecciones, situación que de no ser constantemente cuestionada, se reproduce en cada nueva actividad manteniendo al margen de éstas siempre a las mismas personas. No fue posible realizar el juego y dedicamos todo tiempo del aula a las conversaciones para superar los conflictos.

Acciones: Recordar las reclamaciones surgidas en la clase anterior sobre las personas que no pasaban el balón a las otras y sobre la violencia durante el juego. Debatimos la importancia y el derecho de la participación de todos en la actividad, pues el objetivo de las mismas es posibilitar el aprendizaje de los que ya saben, pero también de los que aún no saben y, por eso, debemos considerar la participación de todos/as. Debatir con los grupos la cuestión del Respeto desde el inicio de la sesión siguiente y para la próxima actividad con juegos en equipos y acompañar de cerca, paso a paso, y mediar la organización de los equipos antes y durante el juego, problematizando frecuentemente con los participantes la situación de juego en que están envueltos y efectuando modificaciones para favorecer la participación equitativa. Dar consejos, orientación y buscar motivar a los niños que demostraban menos habilidad en el juego, a fin de favorecer el aprendizaje de las habilidades necesarias y la participación de las mismas en la actividad.

\section{Sesión 09}

Reflexiones: La división de los equipos no fue realizada de acuerdo con las sugerencias y los grupos terminaron organizándose en equipos no equilibrados como ya había ocurrido. Sin embargo, la problematización permanente durante los juegos hizo emerger los conflictos, principalmente entre equipos, y exigió una solución que significaba efectivamente el compromiso del grupo con una acción que en algunos casos culminó con la reestructuración de la división de los equipos y en otros fueron firmados acuerdos de cooperación entre los niños para que todos pudieran participar. Los niños menos habilidosos participaron con mayor desenvoltura y se arriesgaron más durante las actividades, aunque aún hayan existido diversos conflictos.

Acciones: Dialogar en la próxima sesión sobre el dibujo animado en que aparece un juego de baloncesto con actitudes violentas entre los equipos y situaciones de exclusión de los menos habilidosos buscando relaciones con las situaciones semejantes que vivenciamos en aula.

\section{SITUACIÓN DE APRENDIZAJE 04: HISTORIA EN LA TELA (SESIÓN 10)}

Esta situación de aprendizaje tuvo la duración de una sesión y el objetivo fue presentar con mayor profundidad el tema Historia de los juegos y deportes, abordando aquellos sobre los 
cuales los niños demostraron más interés (Fútbol, Baloncesto, Vóley y Bocha) y dialogando mínimamente sobre el origen y el proceso de transformación de estos. La actividad consistió en la presentación de cuatro videos, ${ }^{6}$ correspondientes a cada una de las modalidades, que fueron asistidos con realización de diversas pausas en las cuales buscábamos problematizar la situación representada en el video, enfatizar alguna información histórica o relacionar la evolución de las modalidades con las actividades realizadas durante las clases. Uno de los videos que más llamó la atención de los niños fue sobre la modalidad Bocha, pues muchos no la conocían y quedaron curiosos sobre el juego, principalmente cuando informamos que el padre de uno de los niños podría venir a la escuela enseñar tal juego.

Otro video bastante debatido fue el dibujo animado que, abordando el origen y transformación del baloncesto, representó en algunos momentos la organización de un equipo presentando escenas "cómicas" con situaciones de marginación y violencia durante la práctica de la citada modalidad. Estas escenas fueron analizadas y comparadas con las situaciones y reclamaciones vivenciadas por el grupo durante la vivencia en sesiones anteriores, llevando al reconocimiento de que estas experiencias, cuando fueron vividas por ellos, no resultaron cómicas ni agradables.

\section{REFLEXIONES SOBRE LA PRÁCTICA Y ACCIONES DESENCADENADAS}

\section{Sesión 10}

Reflexiones: A pesar de una resistencia aparente de algunos niños al saber que la clase de Educación Física de aquel día envolvería solo la presentación de videos, al final tuve la impresión de que les gustó la actividad. Los niños se animaron bastante con el video que trató de la Bocha y preguntaron varias veces si recibiríamos la visita del padre de uno de los niños para enseñarnos el juego. Los diálogos sobre ese video fueron bastante interesantes, muchos niños hicieron comentarios durante el mismo. Durante la conversación sobre el juego de baloncesto fue posible analizar la propia acción de los niños en las sesiones, y ello nos pareció una buena estrategia.

Acciones: Quedamos con los niños en que tendríamos una sesión más con el juego nuevo que íbamos a llevar y que en las dos últimas sesiones las haríamos sobre el juego de la Bocha -en la primera de ellas el padre del niño vendría para enseñarnos el juego-. Para la siguiente sesión definimos con el profesor la realización de un juego africano que los niños aún no conocían, posibilitando su realización antes de la finalización de la intervención con la Bocha. Esta elección se pautó a partir de las sugerencias realizadas por dos niñas durante la investigación, y por nuestro interés en discutir juegos menos conocidos y no transformados en deporte.

Los vídeos exhibidos fueron los siguientes: História do Futebol no Brasil, disponible en: <http://www.youtube.com/ watch?v=PXCgN9RymHg> Voleibol - histórico, disponible en: <http://www.youtube.com/watch?v=EQfNNUdJer0> Pateta - Basquete, disponible en: <http://www.youtube.com/watch?v=6POiulWxJQ0> Bocha do Palestra EC, disponible en: $<$ http://www.youtube.com/watch?v=b4bKoFaPhUI> 


\section{SITUACIÓN DE APRENDIZAJE 05: MY GOD! (AULA 11)}

Para esa actividad fue dedicada una sesión que consistió en vivenciar el juego de origen africano My God ${ }^{7}$, conocer el país en que se originó (Mozambique) y cuestionar por qué este juego no se transformó en deporte. Posteriormente, dialogamos sobre el proceso de padronización y escritura de los deportes, así como su divulgación por el mundo a través de instituciones como asociaciones deportivas y escuelas, con intención de comprender por qué algunos juegos se hicieron deportes conocidos y practicados en todo el mundo y otros no.

En esa actividad el Respeto fue un punto de pauta en el debate sobre falta de compromiso de algunos niños para la realización de las actividades y que originó reclamaciones de otro, pues esas actitudes hacían al equipo perder las partidas. La mediación de los conflictos envolvía trampas, como coger o lanzar el balón estando fuera del espacio determinado por las reglas, lo que también abrió el debate sobre el tema.

\section{REFLEXIONES SOBRE LA PRÁCTICA Y ACCIONES DESENCADENADAS}

\section{Sesión 11}

Reflexiones: Surgieron muchos conflictos en la organización de los equipos y en diversos momentos tuvimos que intervenir mediando en la situación. El gran impasse esta vez ocurrió principalmente porque ninguno de los grupos quería un grupo de niños que, según ellos, no prestaban atención al juego porque solo hablaban y esto implicaba constantemente la derrota del equipo. Después de la mediación en ese conflicto inicial, la realización del juego fue bastante tranquila: todos los niños participaron sin marginación alguna de los niños, la única situación conflictiva surgió con tres chicos que "trampearon" durante el juego y fueron advertidos diversas veces por los compañeros y también por nosotros.

Acciones: Durante la elección de los equipos tuvimos una larga conversación que exigía tratar cuidadosamente las reclamaciones realizadas en la elección que implicaban preocupación por la falta de compromiso de las personas durante las actividades para no perjudicar al grupo. Los chicos del grupo excluido comprendieron que las reclamaciones de los compañeros tenían sentido y aceptaron separarse para no desviar su atención durante la actividad. Al final de la sesión dialogamos sobre el descontento de las personas en relación al comportamiento de los compañeros que estaban "trampeando" y no cumpliendo las reglas acordadas durante el juego, perjudicando al grupo.

\footnotetext{
Juego disponible en el libro Jogos de Moçambique (Prista et al., 1992, p. 42-43), el juego consiste en dos equipos, uno responsable de apilar, un jugador de cada vez, un conjunto de latas (6 a 8) en el formato definido por el grupo (pirámide o torre) en el centro dos líneas paralelas con distancia de aproximadamente $10 \mathrm{~m}$ entre ellas. El otro tiene la función de, posicionados mitad del equipo atrás de cada una de las líneas, darles a los jugadores que están apilando las latas lanzando uno o más balones. El jugador alcanzado es rápidamente sustituido por otro del equipo que continúa su función, cuando se consigue apilar todas las latas, se pasa el pie por encima y se grita "my god!", el jugador que está apilando marca un punto y también es sustituido por un compañero. El juego acaba cuando cada uno de los equipos tiene todos los jugadores quemados, sin embargo posee variaciones con control de tiempo o incluso jugar continuamente alternándose los equipos.
} 


\section{SITUACIÓN DE APRENDIZAJE 06: CONOCIENDO LA BOCHA (SESIONES DE 12 A 13)}

Con dos sesiones, esta situación tuvo el objetivo de fomentar la participación de los familiares en las actividades de la escuela y la puesta en valor del saber de la comunidad, además de profundizar en el conocimiento de las reglas y estructura del juego de Bocha por medio de la vivencia organizada junto al padre de uno de los niños. La actividad fue dividida en dos etapas: la primera consistió en que el padre participante nos presentó los tipos de bolas que eran usadas, explicó el origen y las reglas básicas del juego de Bocha; ${ }^{8}$ $\mathrm{y}$, posteriormente, vivenciamos el juego en el aula de manera adaptada, con colchonetas formando los laterales de las canchas y utilizando balones de plástico o de goma para la realización de la práctica en pequeños grupos, de modo que posibilitara la participación de todos en el corto tiempo de la clase. Ya la segunda etapa consistió en la visita del grupo a un club de la ciudad donde había una cancha oficial de Bocha. Esta visita fue una invitación del padre del chico que personalmente lo agendó con el club y consiguió que la clase fuera guiada por un jugador del espacio. En el espacio del club fuimos recibidos por un campeón de Bocha de la ciudad que nos enseñó más sobre el juego, nos explicó más detalladamente las reglas y nos posibilitó vivenciar el juego con materiales y espacio oficiales para la práctica de la modalidad, además de demostrar algunas jugadas que llamaron la atención de los niños por su precisión y habilidad.

El tema Respeto en esa actividad, además de la cuestión de organización de los equipos, también se relacionó con la participación de los familiares y alumnos de prácticas, así como con el comportamiento y actitudes necesarias para garantizar nuestra seguridad durante el trayecto de la escuela hasta el club, recorrido a pie. También tratamos sobre el respeto necesario al espacio y a las personas que nos iban a recibir en la visita.

\section{REFLEXIONES SOBRE LA PRÁCTICA Y ACCIONES DESENCADENADAS}

\section{Sesión 12}

Reflexiones: La visita del padre fue muy interesante. Los niños prestaron mucha atención y se mostraron curiosos con el tema y bien implicados con la actividad, dando la impresión de haberles gustado bastante. Se quedaron ansiosos por conocer la cancha de Bocha de acuerdo a la posibilidad anunciada por el padre. La poca experiencia de todos los niños con la Bocha dificultó el éxito de los "más habilidosos” y posibilitó experiencias entre algunos de aquellos que no presentaban gran desempeño en otras modalidades. El compromiso, tanto del padre como nuestro, de organizar esa actividad conjunta no fue tarea muy fácil, pues muchas cosas fueron resolviéndose sobre la marcha, sin embargo creemos que eso forma parte de ese proceso de articulación con la comunidad.

\footnotetext{
El padre del chico nos informó que existen registros de juegos semejantes en Egipto y Grecia con más de tres mil años y que, posiblemente, esos juegos dieron origen al juego de Bocha. Comentó que, en esta época, los balones eran hechos de piedras o madera y afirmó que el juego se popularizó en Roma, en Italia, durante el imperio romano con el nombre de Bocce, hoy Bocha, y que a partir de ahí se esparció por el mundo y llegó a Brasil con la inmigración italiana. Básicamente el juego consiste en una disputa de dos equipos, las cuales poseen seis bolas cada uno, con el objetivo de dejar al final de los lanzamientos el mayor número posible de bolas de su equipo próximas al blanco (pequeña bola de acero macizo) colocado a distancia, para eso se puede, inclusive, retirar el blanco o el balón adversario acertándolos con el balón durante el lanzamiento. Después del lanzamiento inicial realizado por cada equipo, los siguientes son determinados por la distancia que cada una mantiene del blanco. Al final vence el equipo que posee las bolas más próximas al blanco.
} 
Acciones: Organizar junto al padre la visita a la cancha de Bocha e invitar familiares y alumnos en prácticas interesados en acompañar la visita. Considerar el uso de juegos poco conocidos para favorecer la participación de todos los niños en las aulas.

\section{Sesión 13}

Reflexiones: Los alumnos en prácticas se interesaron y nos acompañaron en el paseo, así como la hermana de una de las alumnas. Los niños estaban bastante animados con la salida de la escuela. Hasta las ropas estaban diferentes: llevaron accesorios, máquinas fotográficas y bolsas. Una de las madres y un padre demostraron interés en ir, sin embargo dijeron que no podrían por causa del trabajo. Llegamos un poco antes del horario acordado y la cancha aún estaba cerrada, decidimos esperar en la plaza que se encontraba enfrente. Los niños jugaron mucho en aquel espacio. Los alumnos en prácticas y educadores estaban atentos para que no salieran a la calle. Los comportamientos fuera del ambiente escolar se transformaron, las relaciones se establecieron de otra forma, más solidarias, cooperativas y respetuosas, inclusive con la participación de la hermana de una de las niñas que ayudó en el cuidado de los niños. Conversamos entre educadores y alumnos en prácticas sobre la falta de posibilidades de actividades como aquella en el espacio escolar, inclusive la falta de espacio también en las casas y apartamentos que alejan las posibilidades de convivir y juguetear en grupo en el tiempo libre.

\section{CONSIDERACIONES}

La tensa relación entre el perder y el ganar, entre el miedo y el riesgo, entre la devaluación de los niños menos habilidosos y la valorización de los más habilidosos emergió potencialmente durante la intervención realizada. El miedo a la derrota que genera críticas a las personas que yerran y la devaluación del error muchas veces impiden a los niños participar como quisieran, y también ejercitar sus habilidades y creatividad durante la realización de las actividades. Ante esto, la organización de grupos o equipos se reveló como un proceso complejo, sin embargo aprender a convivir se hace imperativo en nuestro contexto social que ha enseñado a los niños a agruparse por interés (o por desinterés) en la habilidad de los compañeros, por género o para intentar protegerse de las "inevitables" reclamaciones y críticas. Se notan las preocupaciones de los niños relacionados con la formación de los grupos que poseen aun otros elementos, pues consideran también el buen desempeño en la evaluación del profesor y revelan una preocupación con la nota atribuida a la personas. Así, se develaron fuertemente las relaciones utilitaristas que tiene la búsqueda de la victoria a cualquier coste, o el miedo, como principal motivación.

El profesor del grupo indicó en una entrevista la percepción de un pequeño avance en el sentido de superación de este tema, según él:

[...] algunos niños... es... cambiaron muy poco en esa relación [...], pero también veo que algunos comienzan un intento de cambio en esas relaciones, de respetar más al otro, entonces cuando hay una discusión, por ejemplo, con esa clase, ahora, en mis sesiones de este año yo percibo que algunas personas se preocupan por resolver los problemas de una forma más tranquila, más sosegada, a través de conversaciones. [...] Me parece que algunos de los alumnos entendieron un poco eso y están intentando promover ese cambio. Tímidamente diría yo, pero me parece que ocurrió alguna cosa con algunos de ellos. ${ }^{9}$

\footnotetext{
Entrevista realizada al profesor de Educación Física del grupo después de la intervención.
} 
Eso nos ayuda a comprender cómo y qué acciones pedagógicas pautadas en los referenciales de la Motricidad Humana pueden hacer contribuciones a las acciones educativas desarrolladas en la Educación Física escolar, pues no existe gesto o acción humana que no se encuentre cargada de intencionalidad y de sentido. Observar las actividades realizadas bajo esa óptica nos permitió develar sentidos y significados en las acciones de los niños del día a día de las clases, y estos, por su parte, nos revelaron la necesidad de considerarlos en la planificación y organización de las sesiones y del tiempo-espacio escolar.

Entendemos que la problematización desarrollada en torno a las actividades fue un punto bastante relevante de la intervención, pues desafiaron tanto a los niños como a nosotros educadores a presentar respuestas a las cuestiones que emergían de esas situaciones, proporcionando un excelente ejercicio de autonomía, de convivencia y de construcción de conocimiento.

Pensando la Motricidad Escolar y el compromiso se destacan elementos fundamentales a la praxis pedagógica, pues toda acción humana expresa intencionalidad, así la acción realizada es expresión del compromiso intencionalmente asumido. En esa perspectiva, cuestionar los sentidos de las acciones, tanto de los niños como nuestras, educadores, fue algo fundamental para comprender y buscar la transformación de las prácticas que habitualmente reproducidas, se hacen alienadas, vacías de sentido. Ante eso la intervención vivenciada por nosotros tuvo contribuciones bastante significativas para la realización de la Motricidad Escolar, pues los diálogos en los procesos de construcción de la autonomía, fueron desafiantes para las personas participantes, ya que los constantes cuestionamientos sobre el sentido de las acciones realizadas en las sesiones los obligaban a identificar el sentido que las mantiene, con compromiso en la transformación de las acciones con las cuales no concordaban y en el cultivo de las acciones que entendían como importantes.

Obviamente, la intervención realizada no responde a todas a las cuestiones, sin embargo nos indicó algunos posibles caminos a continuación. Acordamos que las limitaciones encontradas por nosotros, algunas de las cuales apuntamos aquí, no impiden la realización de un trabajo en la perspectiva propuesta, ellas son inherentes al proceso de pensar y repensar constantemente la práctica. Estas situaciones, lejos de inviabilizar el trabajo pedagógico en la perspectiva asumida, nos indican los próximos pasos.

\section{REFERENCIAS BIBIOGRÁFICAS}

Carmo, C. S. (2013). Motricidade dialógica: compartilhando a construção do conhecimento na educação física escolar (Dissertação de Mestrado em Educação, Programa de Pós-Graduação em Educação). Universidade Federal de São Carlos, São Carlos.

Freire, P. (2005). Pedagogia do oprimido (43 ${ }^{\mathrm{a}}$ ed.). Rio de Janeiro: Paz e Terra.

Jares, X. R. (2002). Conceito de conflito. En X. R. Jares Educação para paz: sua teoria e sua prática ( $2^{\mathrm{a}}$ ed., pp.132-142). Porto Alegre: Artmed.

Merleau-Ponty, M. (2006). A natureza: curso do Collège de France ( $2^{\mathrm{a}}$ ed.). São Paulo: Martins Fontes.

Prista, A., Tembe, M., \& Edmundo, H. (1992). Jogos de Moçambique. Maputo: INEF; Lisboa: CIDAC

Sérgio, M. (1991). Educação física ou ciência da motricidade humana? (2 ed.). Campinas: Papirus.

Sérgio, M. (2003). Para uma nova dimensão do desporto ( $2^{\mathrm{a}}$ ed.). Lisboa: Instituto Piaget.

Sérgio, M., \& Toro, S. (2005). La motricidad humana: un corte epistemológico de la educación física. Consentido, 1, 101-111. 
